\title{
QUANTIFICATION OF FAILURE MECHANISMS IN MODE-I LOADING OF FIBER REINFORCED PLASTICS UTILIZING ACOUSTIC EMISSION ANALYSIS
}

\author{
M. G. R. SAUSE1, T. MÜLLER ${ }^{2}$, A. HOROSCHENKOFF ${ }^{2}$ and S. HORN¹ \\ ${ }^{1}$ University of Augsburg, Institute for Physics, Experimental Physics II, D-86135 \\ Augsburg \\ 2University of Applied Sciences, Department of Mechanical, Automotive and \\ Aeronautical Engineering, D-80335 Munich
}

\section{Abstract}

Acoustic emission signals originating from interlaminar crack propagation in fiber reinforced composites were recorded during double cantilever beam testing. The acoustic emission signals detected during testing were analyzed by feature based pattern recognition techniques. In previous studies it was demonstrated that the presented approach for detection of distinct types of acoustic emission signals is suitable. The subsequent correlation of distinct acoustic emission signal types to microscopic failure mechanisms is based on two procedures. Firstly, the frequency of occurrence of the distinct signal types is correlated to different specimens' fracture surface microstructure. Secondly, a comparison is made between experimental signals and signals resulting from finite element simulations based on a validated model for simulation of acoustic emission signals of typical failure mechanisms in fiber reinforced plastics. A distinction is made between fiber breakage, matrix cracking and interface failure. It is demonstrated, that the feature values extracted from simulated signals coincide well with those of experimental signals. As a result the applicability of the acoustic emission signal classification method for analysis of failure in carbon fiber and glass fiber reinforced plastics under mode-I loading conditions has been demonstrated. The quantification of matrix cracking, interfacial failure and fiber breakage was evaluated by interpretation of the obtained distributions of acoustic emission signals types in terms of fracture mechanics. The accumulated acoustic emission signal amplitudes show strong correlation to the 
mechanical properties of the specimens. Moreover, the changes in contribution to the different failure types explain the observed variation in failure behavior of the individual specimens quantitatively.

Keywords: D: Acoustic Emission, A: Polymer-matrix composites, B: Fracture toughness, B: Delamination, C: Damage mechanics

\section{Introduction}

Fiber reinforced plastics are materials of great interest for modern light-weight engineering since they show extraordinary strength to weight and stiffness to weight ratios. In practice the full potential of carbon fiber reinforced plastics (CFRP) and glass fiber reinforced plastics (GFRP) is rarely used, since reliable failure models for interlaminar failure are still not available $[1,2,3,4,5]$. An improved understanding of interlaminar material failure in fiber reinforced materials will contribute to optimize the mechanical performance of the composite or to reduce its overall weight due to clearly predictable load limits and damage behaviour.

In CFRP and GFRP several microscopic types of failure exist, which result in complex macroscopic failure. Depending on the application and the type of loading the significance of the various failure mechanisms for the composites integrity and stability can change. In order to understand the respective contribution of these failure mechanisms to the failure of the composite it is thus necessary to record their evolution as a function of loading. The microscopic failure mechanisms of interest are fiber breakage, matrix cracking and all sorts of interfacial failure occurring between fiber and matrix (i.e. fiber-matrix debonding, fiber pull-out). Since each of these microscopic failure mechanisms is accompanied by a rapid microscopic displacement of the crack surface, an excitation of an ultrasonic elastic wave occurs inside the material. This 
phenomenon is known as acoustic emission. The detection of the signals at the surface of the solid and their analysis is thus a powerful tool to investigate composite failure. For the current approach the double cantilever beam (DCB) test was chosen to investigate interlaminar failure of fiber reinforced composites under mode-I loading condition.

In general, the acoustic emission signals recorded during failure of composite materials originate from more than one failure type. In the past various authors used acoustic emission analysis to detect the onset and position of failure occurring in fiber reinforced materials $[6,7,8,9,10,30,31,32,33,34]$. Knowing the correlation between the detected acoustic emission signals and a particular failure mechanism, the interpretation of acoustic emission signals is a valuable tool to investigate the integrity of composite structures.

Based on fracture mechanics considerations the driving mechanism of acoustic emission excitation is the movement of the crack surface $[11,12]$. Typical failure mechanisms occurring in DCB testing like matrix cracking, fiber breakage and interfacial failure are illustrated in figure 1. During macroscopic crack propagation under mode-I loading the interlaminar splicing typically causes severe fibre bridging as illustrated in figure 1 . The considered microscopic failure mechanisms exhibit different directions of the crack surface displacements. Dependent of the crack surface movement and the elastic properties of the involved materials (e.g. fiber and resin) this causes formation of characteristic source radiation patterns. This was recently demonstrated utilizing a new acoustic emission source model, which takes into account the inhomogeneity of the elastic properties close to the source [13]. In the case of flat (plate-like) specimens these different source radiation patterns cause excitation and formation of distinct Lamb-wave modes [13, 14, 
$15,16]$. For small specimen geometries and specimen thickness $<5 \mathrm{~mm}$ the ratio between zero-order symmetric $\left(S_{0}\right)$ and zero-order antisymmetric $\left(A_{0}\right)$ Lamb-wave modes can be used to identify the underlying source mechanism. For such thin specimen types, the $\mathrm{S}_{0}$ mode excited by acoustic emission sources propagates dominantly at higher frequencies than the $A_{0}$ mode. This characteristic ratio is also observed in the frequency spectra of the acoustic emission signals. This effect has been reported by various authors, who distinguish between fiber breakage and matrix cracking based on significant contributions at high frequencies (fiber breakage) or low frequencies (matrix cracking) of the acoustic emission signals $[7,9,10,17,18,33,34]$. However, there is a significant shift of the weight of frequency contributions as a function of the distance between the acoustic emission source and the sensor position. Under real experimental conditions the frequency content of the acoustic emission signals depends on velocity dispersion and frequency dependent attenuation. Due to these effects, the acoustic emission signals of different failure types often cannot be separated using fixed frequency parameters. To overcome this problem, parameter based pattern recognition techniques can be applied to form more complex decision criteria to detect and separate clusters of acoustic emission signals $[8,10,17,18,19,20,30,31]$.

To this end we recently proposed a new pattern recognition approach to identify the natural clusters of acoustic emission signals [21]. This method is based on a generalization of the approach introduced by A. Anastassopoulos et al. and utilizes a voting scheme after S. Günter et al. [22, 23]. The application of the proposed cluster validation technique ensures that the most significant clusters of acoustic emission signals are detected with a minimum of initial assumptions on the cluster structure. It is not part of the pattern recognition approach to 
correlate these natural clusters of acoustic emission signals to particular failure types. Such assignment can be achieved by subsequent comparison of the localized source positions to microscopic observations. An alternative approach is the comparison of the experimental acoustic emission signals to respective signals obtained from finite element simulations. A detailed description of the simulation method using the software environment Comsol is beyond the scope of this article, but can be found in $[13,14,15,16]$.

Within $[13,15]$ the modeling of microscopic failure mechanisms like fiber breakage, matrix cracking and interfacial failure is introduced and the excitation of respective Lamb wave modes is discussed. The simulation approach presented does not aim at modeling of crack propagation, but the activity and microstructure of the acoustic emission source. In $[14,16]$ the consequences of different specimen geometries, ply layups and the acoustic emission sensor is introduced. In summary it was demonstrated, that fiber breakage, matrix cracking and interfacial failure result in characteristic ratios of $A_{0}$ and $S_{0} L_{\text {Lamb }}$ wave modes, based on the source orientation and the elastic properties close to the source. Further, the position of the source and the distance to the sensor can cause characteristic shifts in the ratios of $A_{0}$ and $S_{0}$ Lamb wave modes and thus results in broad ranges of frequency parameters correlated with a particular failure mechanism.

In the following we present the experimental setup and give a short introduction to the pattern recognition approach applied to experimental data. Then we present the experimental results and discuss the acoustic emission source identification procedure.

\section{Experimental Setup}


The CFRP specimens investigated were made of the HexPly NCIM 913/35\%/132/T800 prepreg system (T800/913). This prepreg system consists of an unidirectional arrangement of continuous Torayca T800 intermediate modulus carbon fibers and the HexPly 913 epoxy resin. The GFRP specimens were made of the HexPly NVE 913/28\%/192/EC9756 prepreg system, consisting of E-Glass fibers and the HexPly 913 epoxy resin (E-Glass/913). All specimens were manufactured under vacuum conditions using the curing cycle recommended by the material supplier $\left(120^{\circ} \mathrm{C}, 1.5 \mathrm{~h}, 7\right.$ bar). Plates of dimensions $320 \mathrm{~mm} \times 320 \mathrm{~mm} \times 3 \mathrm{~mm}$ (length $\times$ width $\times$ height) with unidirectional $\left[0_{12}\right]$ sym stacking sequence were manufactured. At the medial plane of the specimens a $20 \mathrm{~mm}$ Polytetrafluoroethylene stripe with $50 \mu \mathrm{m}$ thickness was inserted to provide an initiation site for delamination. The test specimens have dimensions of $(240 \pm 2) \mathrm{mm} \times(24 \pm 0.5) \mathrm{mm} \times(3.0 \pm 0.2)$ (length $\times$ width $\times$ height). Five CFRP and five GFRP specimens were tested using a Zwick type 1464 spindle-driven machine with a $500 \mathrm{~N}$ load cell in the experimental setup as shown in figure 2. The displacement rate was set to $10 \mathrm{~mm} / \mathrm{min}$ with continuous crack growth to yield a continuous activity of acoustic emission signals. The crack propagation length was followed visually and was adjusted to be of equal length for CFRP and GFRP specimens, respectively. In order to avoid any influence on delamination propagation by the compressive force of the clamp system the delamination length was chosen as $70 \mathrm{~mm}$ for CFRP and $50 \mathrm{~mm}$ for GFRP specimens. Subsequently, the mode-I interlaminar fracture toughness $\left(G_{\mid c}\right)$ was calculated using the area method following DIN 65563 (method B) applied to the recorded load-displacement curves. 
The acoustic emission signals were recorded using a Physical Acoustic PCl-2 system with 2/4/6 preamplifiers and type WD AE-sensors in linear geometry. The signals were detected with a threshold based triggering using 80/300/1000 as (Peak-Definition-Time/Hit-Definition-Time/Hit-Lockout-Time) at $35 \mathrm{~dB}$ threshold and $40 \mathrm{~dB}$ preamplification and were recorded with an acquisition rate of $10 \mathrm{MS} / \mathrm{s}$. To suppress detection of friction noise between the loading hinge and the specimen and electromagnetic noise a band-pass filter was used ranging from $20 \mathrm{kHz}$ to $1 \mathrm{MHz}$. The acoustic coupling was provided by medium viscosity silicone grease, while the sensors were attached using suitable clamp systems to ensure reproducible mounting pressure. The sensor coupling was then validated by pulsing sensor 1 and measurement of signal amplitudes at sensor 2 and the supplementary sensor 3 . The sound velocity required to calculate the source position (velocity of So-Lamb wave mode) is determined from the difference in arrival time between sensor 2 and 3 and their metric distance. After signal acquisition, the acoustic emission source position was determined based on a hyperbolic localization technique using the sensor arrangement shown in figure 2. Only the signals with valid source positions (i.e. localized within the specimen) of sensor 1 and 2 were used for further analysis. In order to separate multiple hits within one recorded signal, a postprocessing step with 10/100/1000 (Peak-Definition-Time/Hit-Definition-Time/Hit-LockoutTime) was used. Subsequently the Fast Fourier Transformation $\tilde{U}(f)$ of the signals was calculated and the features listed in table 1 were extracted from the AE-signals and were used for the pattern recognition method described in section 3 . To visualize the time-frequency content of the signals, continuous wavelet transformation using the Gabor-Wavelet included in the software package AWARE++ was used [29]. 


\section{Pattern recognition methodology}

Since the complete pattern recognition method is comprehensively described in [21] only a short review will be given in the following. The task of the pattern recognition approach is the identification of natural clusters of signals. These clusters of signals are than correlated to physical phenomena like particular failure mechanisms by additional investigations using microscopy or comparison to results of finite element simulations.

Technically the approach uses an evaluation of all partitions achieved by subset formation of suitable preselected features. This is done in an exhaustive search procedure, which investigates all subset feature combinations of table 1. For each feature combination, all partitions for $2,3, \ldots, 10$ clusters are evaluated to detect the associated number of natural clusters. This evaluation is performed following the cluster validity index method introduced by A. Anastassopoulos et al. [22]. The voting scheme of S. Günter et al. was applied [23]. Finally, the results of all subset feature combinations are again evaluated to yield the global optimal partition and the associated feature combination. The obtained clusters of acoustic emission signals were found to be highly correlated to the occurrence of particular acoustic emission source types $[13,14,16]$. It is worth noting, that in general no unique $A E$-signal feature combination is expected, which is suitable to distinguish between the particular failure mechanisms under all experimental conditions. Instead the feature combination is inevitably linked to the chosen geometry of the specimen (in particular the thickness) and the stacking sequence of the plies. This is a direct consequence of the dependency of Lamb-wave propagation on the elastic properties and the thickness of the plate structure. Further, the dispersive propagation of Lamb- 
waves causes loss of distinguishability with increasing source-sensor distance. This translates in broader ranges of the individual failure mechanisms feature values and thus causes increasing overlap of cluster structures. For the current source-sensor distances below $100 \mathrm{~mm}$, the classification error based on the overlap of clusters was estimated in [21] to be in the range of $4 \%$ to $5 \%$. Thus further increase of the mean source-sensor distance would require enhanced pattern recognition techniques or selection of different features to distinguish between particular failure mechanisms within this accuracy.

\section{Results and Discussion}

\subsection{Microscopic observations and mechanical properties}

As expected from the loading condition most of the failure in the specimens is observed in the medial plane of the specimen. Microscopically, failure consists

of matrix cracking, interfacial failure and fiber breakage due to bending forces of the beams. Scanning electron microscopy images of representative fracture surfaces of CFRP specimens are shown in figure 3a-d.

The measured interlaminar fracture toughness values of CFRP and GFRP specimens are summarized in table 2. The GFRP specimens show higher $\mathrm{G}_{l_{c}}$ values than the CFRP specimens, with lesser scattering.

The fracture surfaces of the specimen were investigated by scanning electron microscopy. The morphology of the fracture surface can be subdivided into different regions. Firstly, there are rough-structured regions (figures 3a) with a high amount of interlaminar splicing and numerous fiber breakages. Secondly, there are smooth regions, showing almost no interlaminar splicing (figures 3b). Details of the fracture surface (matrix cracking, interface failure, fiber breakage) are shown in figures $3 \mathrm{c}$ and $3 \mathrm{~d}$. Specimens with high $\mathrm{Gl}_{\mathrm{lc}}$ values are dominated 
by areas with rough microstructure (figures $3 a$ ), while specimens with low $\mathrm{G}_{\mathrm{lc}}$ values are dominated by smooth regions (figures $3 b$ ).

\subsection{Comparison between pattern recognition and finite element simulation results}

The pattern recognition approach described in section 3 detects an optimal separation of the acoustic emission signals into three clusters for the feature combination Peak Frequency, Weighted Peak Frequency, Partial Power 1, Partial Power 2 and Partial Power 4 as defined in table 1. In the following the detected clusters are correlated with their respective source mechanisms. This was achieved by comparison to results of finite element simulations.

In figure 4 continuous wavelet transformation results of representative signals of each cluster are shown. Using the hyperbolic localization technique the $\mathrm{x}$ coordinate of the source position was determined. The calculated x-positions and the symmetric position within the medial plane $(y, z)=(0,0) \mathrm{mm}$ of the specimens were used as source position for the finite element simulations of signals resulting from matrix cracking, interfacial failure and fiber breakage (see figure 5). As discussed in $[13,15,21]$ the different failure mechanisms result in different intensities of symmetric and antisymmetric Lamb-wave modes, which contribute to the frequency range above $400 \mathrm{kHz}$ at the beginning of the signal (So-mode) and below $400 \mathrm{kHz}$ after $0.02 \mathrm{~ms}$ (A0-mode). Based on these intensity contributions, a first assignment can be made. Signals of cluster 0 (fig. 4a) agree well with simulated signals of matrix cracking (fig. $4 d$ ), signals of cluster 1 (fig. 4b) show similar intensities as simulated signals of interfacial failure (fig. 4e) and signals of cluster 2 (fig. 4c) agree in their high So-mode intensity with simulated signals of fiber breakage (fig. 4f). 
During loading of the specimen the crack propagates along the x-direction, which causes changes of the source-sensor distance (see figure 5). Since attenuation and dispersion cause changes of the intensity of the different Lambwave modes during signal propagation, the effect of different source-sensor distances has to be taken into account.

Consequently, to compare experimental signals and simulated signals on a quantitative basis, signals originating from matrix cracking, interfacial failure and fiber breakage were simulated at different source-sensor distances. Reflecting experimental conditions, the position of the sensor was kept constant, while the ( $\left.\mathrm{x}_{0}, \mathrm{y} 0\right)$-position of the AE-source was varied at constant zo-position. The same features as given in table 1 were extracted from the simulated AE-signals. A comparison of the cluster distributions of experimental signals and simulated signals is shown in figure 6 in a scatterplot of Partial Power 4 over Weighted Peak-Frequency. Clearly, the value ranges of cluster 0 and the simulated signals of matrix cracking coincide well. The value ranges of simulated signals of interfacial failure and fiber breakage agree with those of cluster 1 and cluster 2 , respectively.

\subsection{Quantification of acoustic emission signals}

After assignment of distinct signal types (clusters) to respective failure mechanisms we compare AE-signal energies to energetic quantities derived from fracture mechanics approaches. As predicted by the generalized theory of acoustic emission [24, 25] and also demonstrated within finite element simulations [16], the amplitude $U_{A E}$ of an acoustic emission signal detected at distance $r$ is proportional to the vibrating crack volume $\Delta V$ : 


$$
U_{A E}=\beta_{U}\left(c_{L}^{2}\right) \cdot \frac{\Delta V}{r}
$$

The proportionality constant $\beta_{U}$ is a linear function of the cracking materials squared longitudinal sound velocity $c_{L}^{2}$ and the orientation of the crack surface movement (not taken into account in equation (1)).

For the case of stable mode-I crack growth a relation similar to equation (1) can be obtained for the energy of an acoustic emission signal $W_{A E}$ using the fracture toughness $G_{I c}$ and the crack surface area $\Delta A$ (see $\left.[16,26]\right)$ :

$W_{A E}=\beta_{W}\left(c_{L}^{2}\right) \cdot G_{I c} \Delta A$

Note that equation (2) uses a different proportionality constant $\beta_{W}$.

As a consequence of equations (1) and (2) the acoustic emission energy release in fiber reinforced composites is expected to be proportional to the mechanical energy release. Each failure mechanism shows a characteristic proportionality constant. Consequently, the acoustic emission energy $W_{A E}$ of each failure type should be compared to the respective fracture energies of the associated failure type, as done in [26]. However, in the current setup it is difficult to measure contributions of the different failure mechanisms to the total fracture energy independently.

In figure 7 the accumulated signal amplitudes of the identified failure mechanisms are compared to the respective total fracture energy as derived from the load-displacement curves of each experiment. Based on fracture mechanics considerations, continuous crack growth would result in stable contributions of the different failure mechanisms during macroscopic crack propagation. Thus a linear correlation between the total fracture energy and the accumulated signal amplitudes for each failure mechanisms is expected. As 
shown in figure 7 this correlation is fulfilled for interfacial failure and fiber breakage. However, larger deviations from linear behaviour are observed for matrix cracking. The scatter can be attributed to additional matrix cracks, which might occur ahead and past the macroscopic crack tip. These cracks are expected to show only little correlation to the total fracture energy as measured in the current setup.

The three failure types show different proportionality constants, which are attributed to the difference in the average oscillating crack volume $\Delta V$ and the different crack surface displacements. However, the variation in the proportionality constants is mainly due to the different frequency of occurrence of the different failure mechanisms. In particular, fiber breakage occurs rarely, resulting in smaller accumulated signal amplitudes than observed for matrix cracking and interface failure. To further investigate the correlation between acoustic emission signal parameters and fracture mechanics quantities, other possibilities to quantify and visualize the occurrence of certain failure types are demonstrated in figure 8a-c.

Usually in DCB testing the fracture mechanics quantity of interest is the fracture toughness value, which was chosen as the $x$-axis in the following. Since the absolute number of acoustic emission signals is affected by the absolute length of crack propagation and the signal acquisition settings, absolute values are often error-prone. Here we introduce normalized quantities to interpret the occurrence of particular failure mechanisms. We define the relative number of signals $N_{r e l, j}$ and the relative amplitude of signals $U_{r e l, j}$, defined for the $j$-th of $J_{\text {tot }}$ failure mechanisms with $N_{j}$ signals as:

$$
N_{r e l, j}=\frac{N_{j}}{N_{t o t}}
$$


$U_{r e l, j}=\sum_{i=1}^{N_{i}} U_{i j} \cdot\left(\sum_{j=1}^{J_{t e n}} \sum_{i=1}^{N_{j}} U_{i j}\right)^{-1}$

Thus $N_{r e l, j}$ and $U_{r e l, j}$ express the contribution of a particular failure type relative to all recorded signals $N_{\text {tot }}$ with accumulated signal amplitudes $\sum_{j=1}^{J_{t o t}} \sum_{i=1}^{N_{j}} U_{i j}$. In addition, the average signal amplitude $\left\langle U_{j}\right\rangle$ of the $j$-th failure mechanism is defined as:

$\left\langle U_{j}\right\rangle=\sum_{i=1}^{N_{j}} U_{i j} \cdot\left(N_{j}\right)^{-1}$

In figures 8a-c results of all experiments on CFRP and GFRP are plotted. The two specimen types are easily distinguished by their fracture toughness values which are $<600 \mathrm{~J} / \mathrm{m}^{2}$ for CFRP specimens and $>600 \mathrm{~J} / \mathrm{m}^{2}$ for GFRP specimens. For CFRP specimens the contributions of interface failure increase from $45 \%$ to $85 \%$, while the contributions of matrix cracking decrease with increasing Gı values, respectively. The contribution of fiber breakage signals is independent of fracture toughness and below $5 \%$. In contrast, no significant tendency is observed for GFRP specimens. This is most likely owed to the fact, that a correlation between fracture mechanics properties and acoustic emission signals is better reflected by energetic signal features and not just the number of acoustic emission signals.

Such an energetic feature is the relative amplitude of signals shown in figure $8 \mathrm{~b}$. Indeed, it reveals a clear correlation to the fracture toughness value. For both material types specimens with increased fracture toughness values show an increase of the contribution of interfacial failure and a respective decrease of matrix cracking. Still the contribution of fiber breakage is nearly constant. The results presented in figure $8 \mathrm{a}$ and $8 \mathrm{~b}$ are consistent with the microscopic observations shown in figure 3 . Here, specimens with high fracture toughness 
values showed large areas of interlaminar splicing, which can be caused by changes in the local interfacial bonding strength or variations in the microscopic loading conditions. In contrast, specimens with low fracture toughness values show large regions of smooth crack surface areas. In terms of acoustic emission source types this can be understood as matrix cracking with lesser occurrence of interfacial failure than for rough fracture surfaces. Fiber breakage in turn was rarely observed in the microscopic investigations, which is consistent with the low number of signals associated with this failure mechanism.

It is worth pointing out, that extrapolation of the data points of figure $8 \mathrm{~b}$ to small fracture toughness values intersects at $50 \%$ relative amplitude contributions within the margin of error, for both CFRP and GFRP specimens. An equal contribution of both failure types would correspond to energetic equality of crack growth within the matrix material and along the interface. The correlation of the Gıc value determined from the abscissa of the intersection to the fracture toughness of neat resin shall be addressed in further investigations.

The average signal amplitude was quantified and plotted in dependence of the fracture toughness value in figure $8 \mathrm{c}$. Within the margin of error matrix cracking and interfacial failure show constant average signal amplitudes. This is indicative of continuous crack growth conditions, as expected for this type of experiment. It is worth noting, that the absolute number of signals originating from fiber breakage scatters drastically (between 58 and 413) depending on the specimen. The error in the average signal amplitude is higher, due to the fact, that the absolute number of signals is one to two orders of magnitude less than for matrix cracking and interfacial failure. 
Based on equation (2) the signal energy of fiber breakage can be estimated based on the fiber cross-sections and the respective mode-l fracture toughness. The cross-section was found by microscopic investigations to be of $(33.6 \pm 1.4)$ $\mu \mathrm{m}^{2}$ for glass fibers and $(21.2 \pm 0.2) \mu \mathrm{m}^{2}$ for carbon fibers. Literature values for the fracture toughness are $0.9 \mathrm{MPam}^{1 / 2}$ for E-Glass [27] and $1.0 \mathrm{MPam}^{1 / 2}$ for T800 carbon fibers [28]. Taking into account the different longitudinal sound velocities $c_{L}$ the expected acoustic emission signal energy would be proportional to $(0.0126 \pm 0.0031) \mathrm{Nm}^{3} / \mathrm{s}^{2}$ for glass fibers and $(0.0131 \pm 0.0005)$ $\mathrm{Nm}^{3} / \mathrm{s}^{2}$ for carbon fibers. Thus single fiber breakage of E-glass fibers and T800 carbon fibers cause nearly the same acoustic emission signal amplitude. This is consistent with the observation, that the average signal amplitude of fiber breakage signals is similar for carbon fibers and glass fibers, which is shown in figure 8c. However, the absolute voltage shown in figure 8c depends on the mean source-sensor distance, the crack surface movement direction and the signal acquisition equipment. Since the same setup was used for all experiments, we do not expect these parameters to have significant influence on the results.

\section{Conclusion}

In the present investigation it was demonstrated how acoustic emission measurements can be used to assess the occurrence of microscopic failure mechanisms in fiber reinforced plastics. It was demonstrated how the quantification of those $\mathrm{AE}$-signals can be used to interpret the variation of mechanical properties typically observed during mechanical testing of composites in DCB loading conditions. The distinction of different AE-signal types is based on pattern recognition techniques as described in [21]. The 
correlation to particular source mechanisms is achieved by comparison to results of finite element simulation as introduced in $[13,14,15,16]$ and was also confirmed by respective microscopic investigations.

In summary, the presented method is able to distinguish between the occurrence of matrix cracking, interfacial failure and fiber breakage in double cantilever beam testing and can be used to quantify these failure mechanisms, respectively. Also the current configuration could be understood as proof of principle to adopt the proposed method to investigate different loading conditions, specimen geometries, stacking sequences and materials.

\section{Literature}

[1] Z. Hashin. Analysis of cracked laminates - A variational approach. Mechanics of Materials, 4, 121-136, 1985.

[2] J. A. Nairn. The Strain Energy Release Rate of Composite Microcracking: A Variational Approach. Journal of Composite Materials, 23, 1106-1129, 1989.

[3] J. A. Nairn, S. Hu. Matrix Microcracking. Damage Mechanics of Composite Materials, 9, 187243, 1994.

[4] L. J. Hart-Smith. Comparison between theories and test data concerning the strength of various fibre-polymer composites. Composites Science and Technology, $62: 12-13,1591-1618,2002$

[5] M. J. Hinton, A. S. Kaddour, P. D. Soden. A further assessment of the predictive capabilities of current failure theories for composite laminates: comparison with experimental evidence. Composites Science and Technology, 64:3-4, 549-588, 2004.

[6] M. Giordano, A. Calabro, C. Esposito, A. D'Amore, L. Nicolais. An acoustic-emission characterization of the failure modes in polymer-composite materials. Composites Science and Technology, 58, 1923-1928, 1998.

[7] J. Bohse. Acoustic emission characteristics of micro-failure processes in polymer blends and composites. Composites Science and Technology, 60, 1213-1226, 2000.

[8] S. Huguet, N. Godin, R. Gaertner, L. Salmon, D. Villard. Use of acoustic emission to identify damage modes in glass fibre reinforced polyester. Composites Science and Technology, 62, 1433-1444, 2002.

[9] W. Haselbach, B. Lauke. Acoustic emission of debonding between fibre and matrix to evaluate local adhesion. Composites Science and Technology, 63:15, 2155-2162, 2003. 
[10] C. R. Ramirez-Jimenez, N. Papadakis, N. Reynolds, T. Gan, P. Purnell, M. Pharaoh. Identification of failure modes in glass/polypropylene composites by means of the primary frequency content of the acoustic emission event. Composites Science and Technology, 64, 1819-1827, 2004.

[11] G. Eason, J. Fulton, I. N. Sneddon. The Generation of Waves in an Infinite Elastic Solid by Variable Body Forces. Philosophical Transactions of the Royal Society A, 248, 575-607, 1956.

[12] L. B. Freund. The initial wave front emitted by a suddenly extending crack in an elastic solid. Journal of Applied Mechanics, 39, 601-602, 1972.

[13] M. G. R. Sause, S. Horn. Simulation of acoustic emission in planar carbon fiber reinforced plastic specimens. Journal of Nondestructive Evaluation, 29:2, 123-142, 2010.

[14] M. G. R. Sause, S. Horn. Influence of Specimen Geometry on Acoustic Emission Signals in Fiber Reinforced Composites: FEM-Simulations and Experiments. 29th European Conference on Acoustic Emission Testing, Vienna, Austria, 2010.

[15] M. G. R. Sause, S. Horn. Simulation of Lamb Wave Excitation for Different Elastic Properties and Acoustic Emission Source Geometries. Journal of Acoustic Emission 28, 109-121, 2010.

[16] M. G. R. Sause. Identification of failure mechanisms in hybrid materials utilizing pattern recognition techniques applied to acoustic emission signals. PhD-Thesis, mbvVerlag, Berlin, 2010.

[17] A. Marec, J.-H. Thomas, R. Guerjouma. Damage characterization of polymer-based composite materials: Multivariable analysis and wavelet transform for clustering acoustic emission data. Mechanical Systems and Signal Processing, 22, 1441-1464, 2008.

[18] X. Li, C. Ramirez, E. L. Hines, M. S. Leeson, P. Purnell, M. Pharaoh. Pattern Recognition of Fiber-reinforced Plastic Failure Mechanism using Computational Intelligence Techniques. Neural Networks, pages 2340-2345. IEEE World Congress on Computational Intelligence, 2008.

[19] T. Philippidis, V. Nikolaidis, A. Anastassopoulos. Damage Characterisation of C/C laminates using Neural Network Techniques on AE signals. NDT\&E International, 31, 329-340, 1998.

[20] N. Ativitavas, T. Pothisiri, T. J. Fowler. Identification of Fiber-reinforced Plastic Failure Mechanisms from Acoustic Emission Data using Neural Networks. Journal of Composite Materials, 40:3, 193-226, 2006.

\section{[21] Submitted to Pattern Recognition Letters}

[22] A. A. Anastassopoulos, T. P. Philippidis. Clustering Methodology for the Evaluation of Acoustic Emission from Composites. Journal of Acoustic Emission, 13, 11-21, 1995.

[23] S. Günter, H. Bunke. Validation indices for graph clustering. Pattern Recognition Letters, 24, 1107-1113, 2003.

[24] M. Ohtsu, K. Ono. A generalized theory of acoustic emission and Green's function in a half space. Journal of Acoustic Emission, 3, 27-40, 1984. 
[25] M. Ohtsu, K. Ono. The generalized theory and source representation of acoustic emission. Journal of Acoustic Emission, 5, 124-133, 1986.

[26] M. G. R. Sause, F. Haider, S. Horn. Quantification of metallic coating failure on carbon fiber reinforced plastics using acoustic emission. Surface and Coatings Technology, 204:3, 300-308, 2009.

[27] C. R. Kurkjian. Mechnical properties of phosphate glasses. Journal of NonCrystalline Solids, 263-264, 207-212, 2000.

[28] B. Zhang, Y. Wu. Experiment research for fracture toughness of PAN-based carbon fibers. Key Engineering Materials, 462-463, 1361-1366, 2011.

[29] M. G. R. Sause. AWARE++ Software manual Rev. 1.3. http://www.physik.uniaugsburg.de/exp2/downloads.de.html, 2009.

[30] V. Kostopoulos, P. Tsotra, P. Karapappas, S. Tsantzalis, A. Vavouliotis, T. H. Loutas, A. Paipetis, K. Friedrich, T. Tanimoto. Mode I interlaminar fracture of CNF or/and PZT doped CFRPs via acoustic emission monitoring. Composites Science and Technology, 67, 822-828, 2007.

[31] V. Kostopoulos, P. Karapappas, T. Loutas, A. Vavouliotis, A. Paipetis, P. Tsotra. Interlaminar Fracture Toughness of Carbon Fibre-Reinforced Polymer Laminates With Nano- and Micro-Fillers. Strain, 47, 269-282, 2011.

[32] J. Bohse. Acoustic Emission Examination of Polymer-Matrix Composites. Journal of Acoustic Emission, 22, 208-223, 2004.

[33] J. Bohse, J. Chen. Acoustic Emission Examination of Mode I, Mode II and MixedMode I/II Interlaminar Fracture of Unidirectional Fiber-Reinforced Polymers, Journal of Acoustic Emission, 19, 1-10, 2001.

[34] M. Eaton, K. Holford, C. Featherston, R. Pullin. Damage in Carbon Fibre Composites: The Discrimination of Acoustic Emission Signals Using Frequency, Journal of Acoustic Emission, 25, 140-148, 2007.

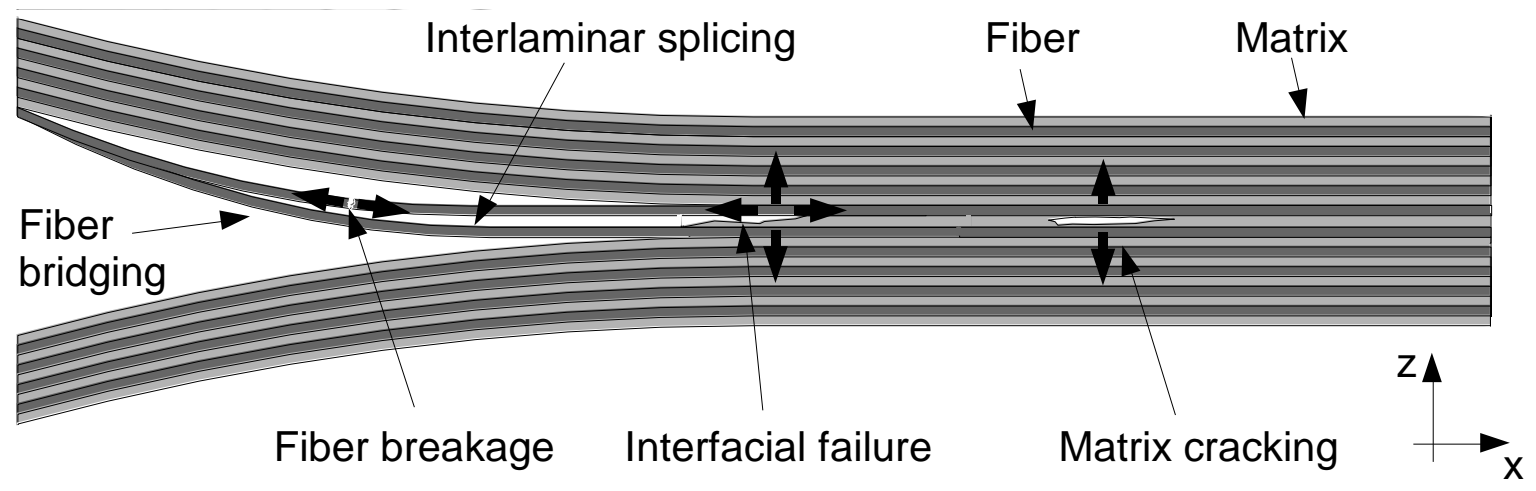

Figure 1: Scheme of various failure mechanisms in fiber reinforced plastics under mode-I loading conditions in DCB testing. 


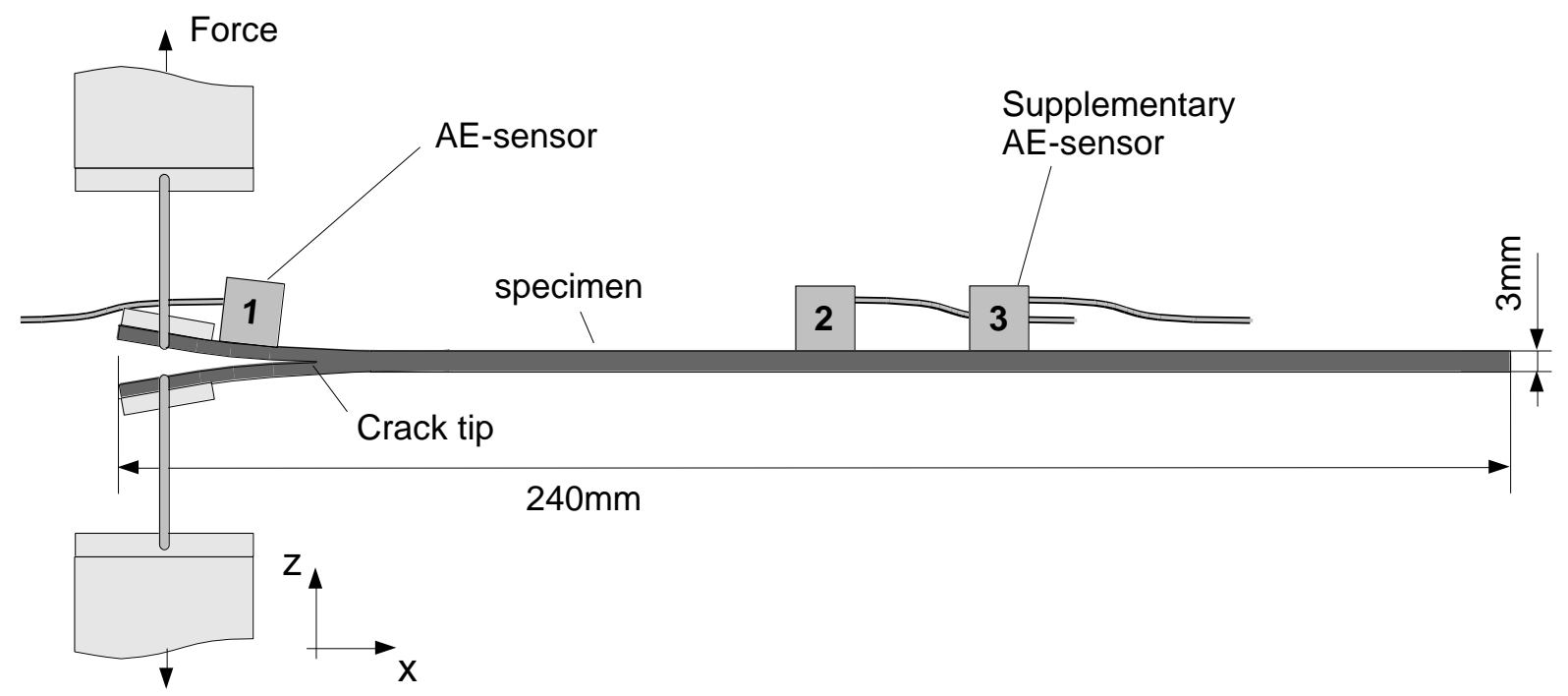

Figure 2: Loading configuration for double cantilever beam testing including positions of AE-sensors.
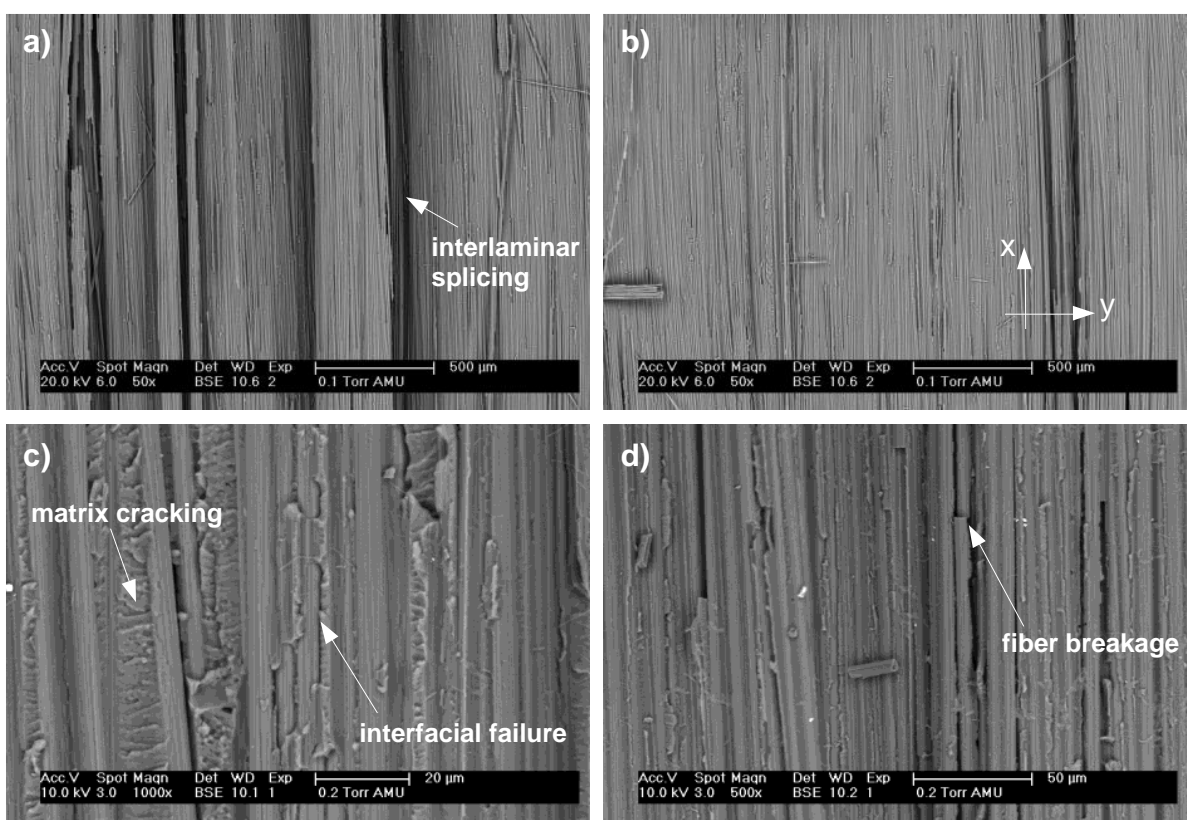

Figure 3: Scanning electron microscopy images of fracture surface in CFRPspecimens within test region (top view). Image of rough regions (a), smooth regions (b) and details of fracture surface $(c, d)$.
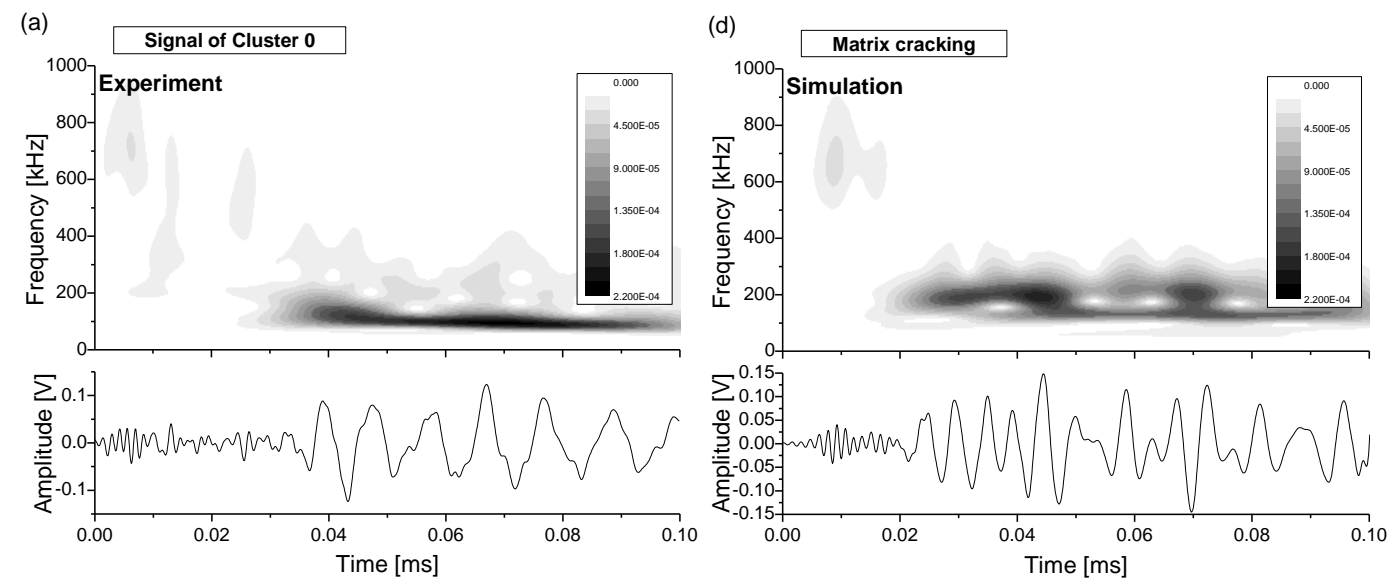
(b)

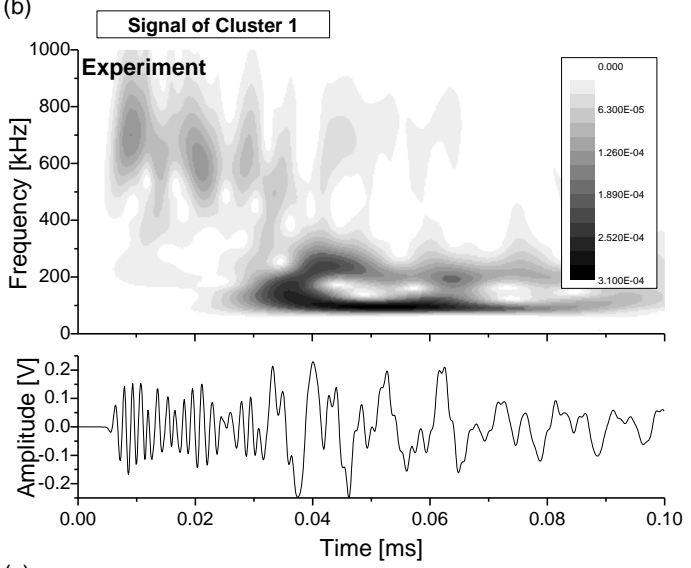

(c)
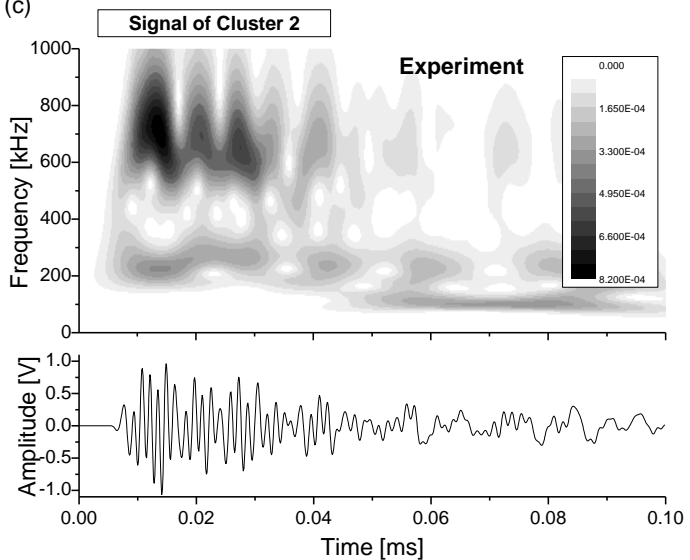

(e)

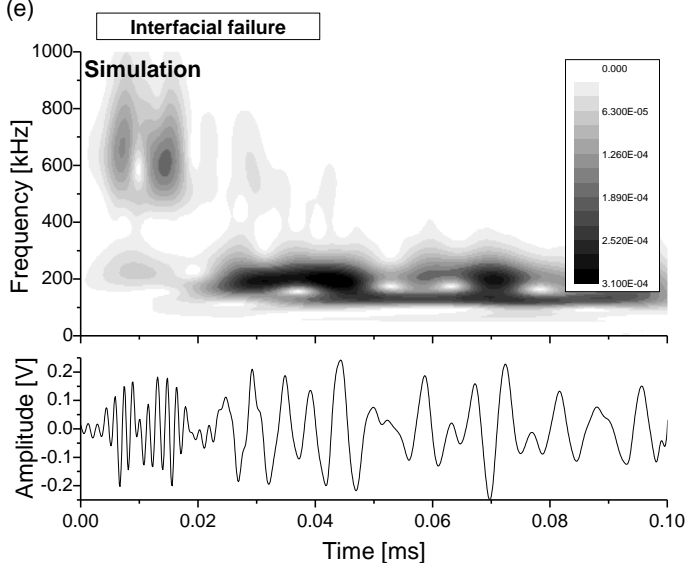

(f)
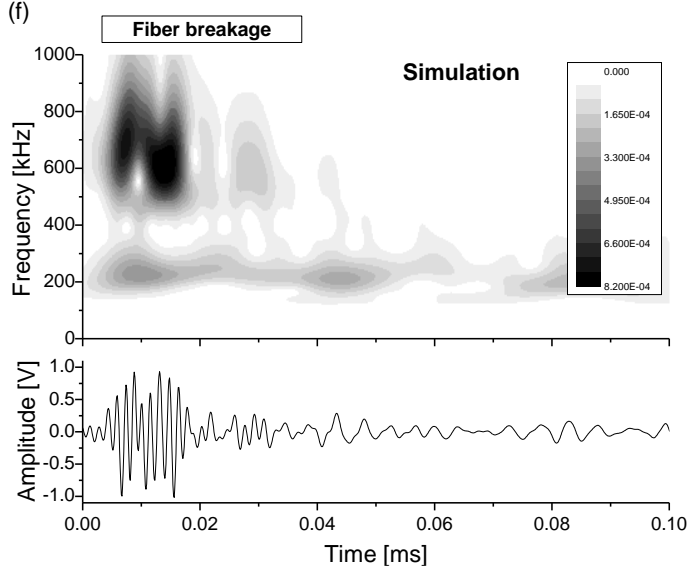

Figure 4: Wavelet spectra of single representative AE-signal of each cluster (ac) and wavelet spectra of simulated AE-signal of matrix cracking (d), interfacial failure (e) and fiber breakage (f).

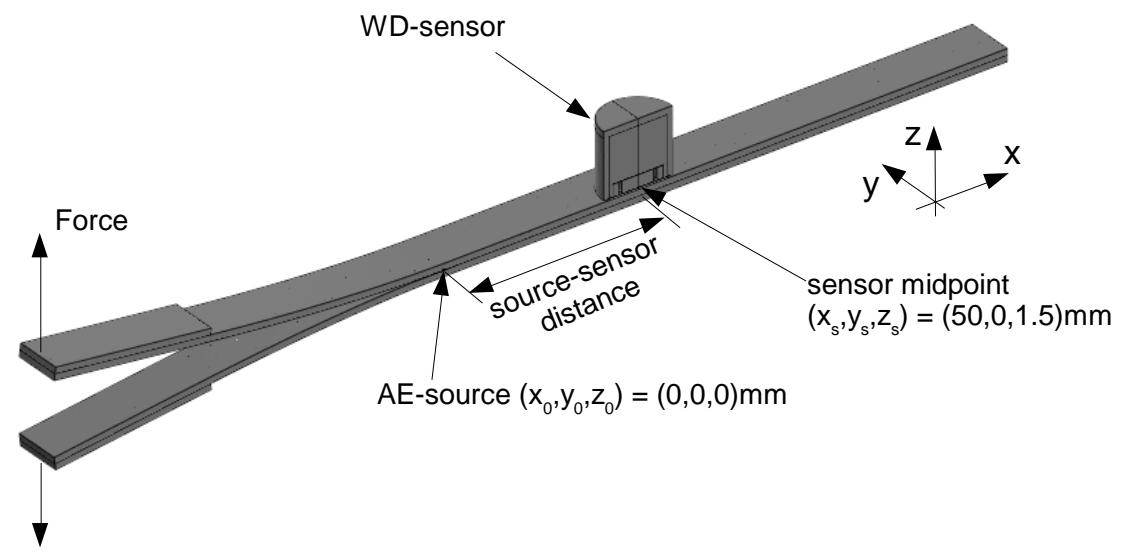

Figure 5: FEM geometry (half-volume) for simulation of acoustic emission signals in DCB-specimens. 


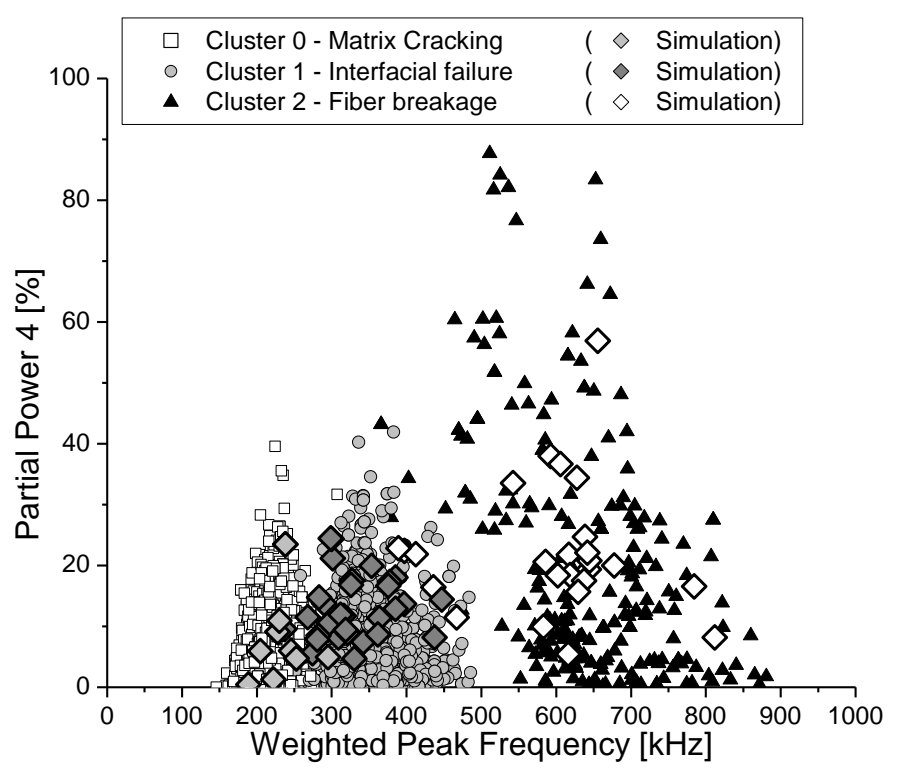

Figure 6: Comparison of experimental signal clusters from pattern recognition approach (specimen CFRP_001) with signal clusters of simulated signals of matrix cracking, interfacial failure and fiber breakage at different source-sensor distances.

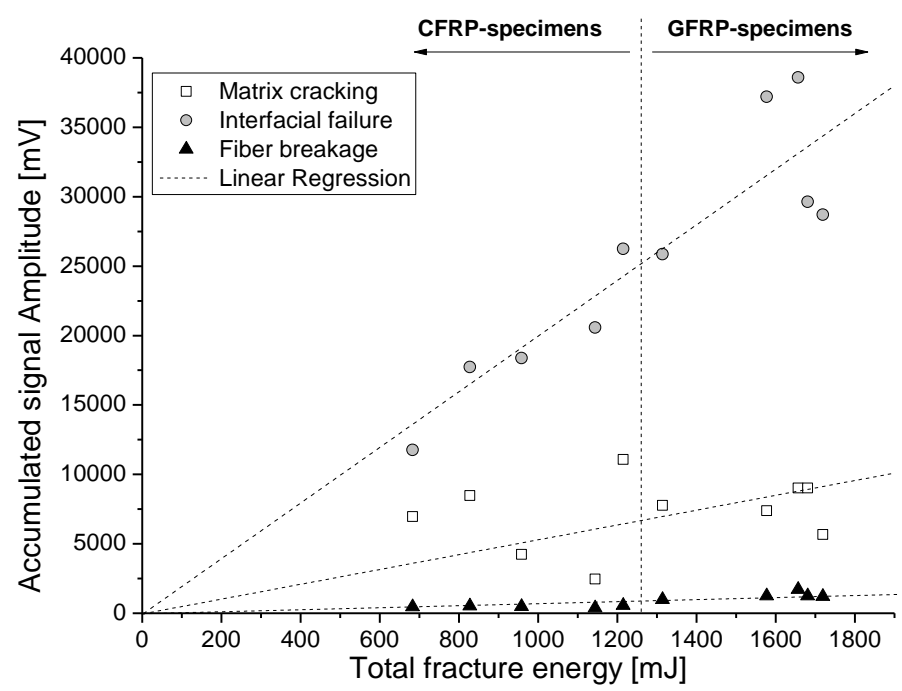

Figure 7: Comparison of accumulated AE-signal amplitudes of different failure mechanisms with total fracture energy for all CFRP and GFRP specimens. 

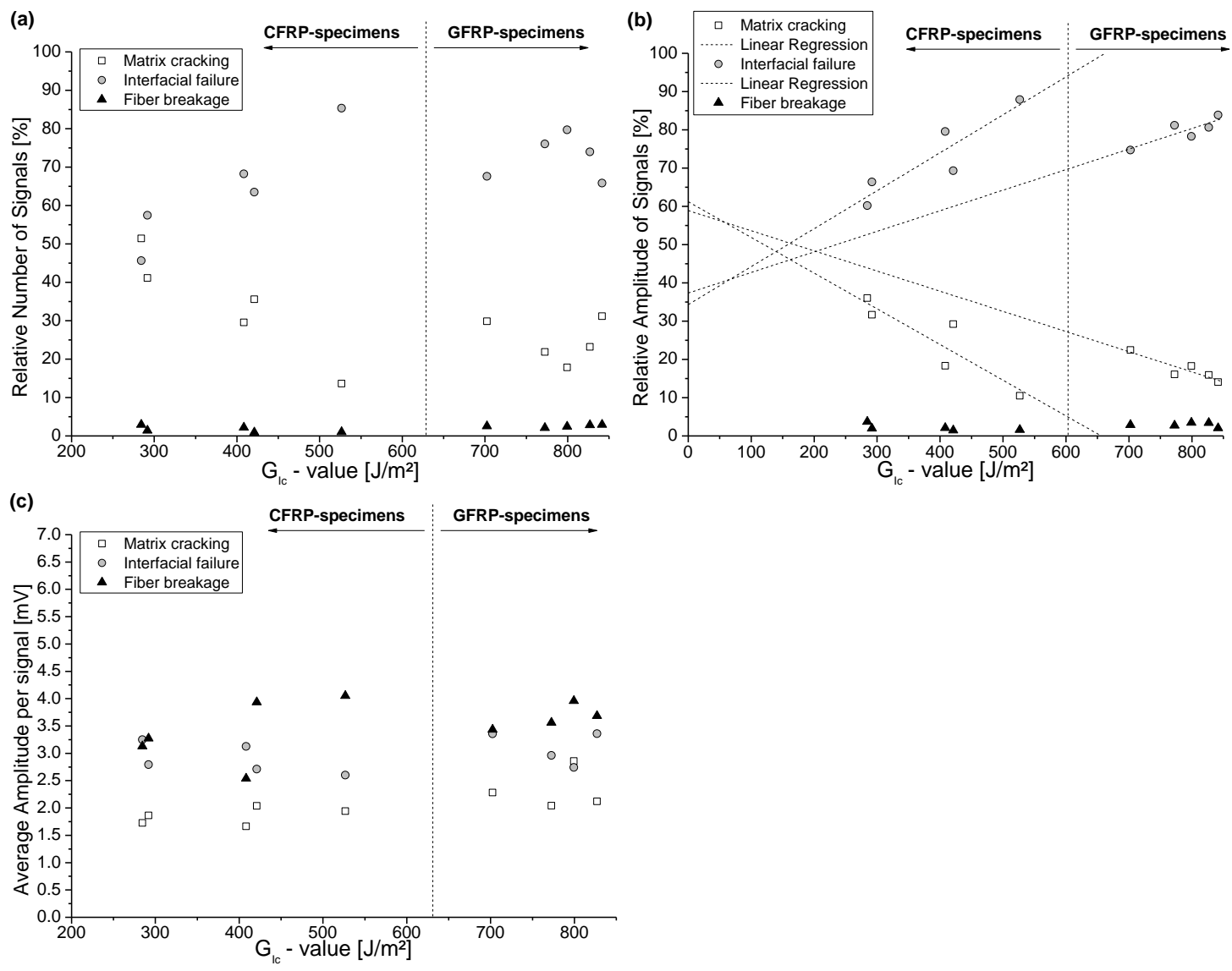

Figure 8: Quantification of relative number of signals (a), relative amplitude of signals (b) and average amplitude per signal (c) in dependence of fracture toughness value for all CFRP and GFRP DCB-specimens. 


\begin{tabular}{|c|c|}
\hline Feature & Definition \\
\hline Average Frequency [Hz] & $\langle f\rangle=N_{A E} / t_{A E}$ \\
\hline Reverberation Frequency [Hz] & $f_{\text {rev }}=\frac{N_{A E}-N_{\text {peak }}}{t_{A E}-t_{\text {peak }}}$ \\
\hline Initiation Frequency [Hz] & $f_{\text {init }}=\frac{N_{\text {peak }}}{t_{\text {peak }}}$ \\
\hline Peak Frequency [Hz] & $f_{\text {peak }}$ \\
\hline Frequency Centroid [Hz] & $f_{\text {centroid }}=\frac{\int f \cdot \tilde{U}(f) d f}{\int \tilde{U}(f) d f}$ \\
\hline Weighted Peak-Frequency [Hz] & $\left\langle f_{\text {peak }}\right\rangle=\sqrt{f_{\text {peak }} \cdot f_{\text {centroid }}}$ \\
\hline Partial Power 1-6 [\%] & $\begin{array}{l}\int_{f_{1}}^{f_{2}} \tilde{U}^{2}(f) d f \int_{0 \mathrm{kHz}}^{1200 \mathrm{kHz}} \tilde{U}^{2}(f) d f \\
\text { Partial Power 1: } \mathrm{f}_{1}=0 \mathrm{kHz} ; \mathrm{f}_{2}=150 \mathrm{kHz} \\
\text { Partial Power 2: } \mathrm{f}_{1}=150 \mathrm{kHz} ; \mathrm{f}_{2}=300 \mathrm{kHz} \\
\text { Partial Power 3: } \mathrm{f}_{1}=300 \mathrm{kHz} ; \mathrm{f}_{2}=450 \mathrm{kHz} \\
\text { Partial Power 4: } \mathrm{f}_{1}=450 \mathrm{kHz} ; \mathrm{f}_{2}=600 \mathrm{kHz} \\
\text { Partial Power 5: } \mathrm{f}_{1}=600 \mathrm{kHz} ; \mathrm{f}_{2}=900 \mathrm{kHz} \\
\text { Partial Power 6: } \mathrm{f}_{1}=900 \mathrm{kHz} ; \mathrm{f}_{2}=1200 \mathrm{kHz}\end{array}$ \\
\hline
\end{tabular}

Table 1: Acoustic emission signal features used for pattern recognition.

\begin{tabular}{ll}
\hline Specimen & G $_{\mathrm{lc}-\text { value }}\left[\mathbf{J} / \mathbf{m}^{2}\right]$ \\
\hline CFRP_001 & $284 \pm 4$ \\
CFRP_002 & $292 \pm 4$ \\
CFRP_003 & $421 \pm 6$ \\
CFRP_004 & $527 \pm 5$ \\
CFRP_005 & $408 \pm 3$ \\
CFRP average & $386 \pm 101$ \\
\hline GFRP_001 & $799 \pm 7$ \\
GFRP_002 & $827 \pm 8$ \\
GFRP_003 & $842 \pm 9$ \\
GFRP_004 & $702 \pm 7$ \\
GFRP_005 & $773 \pm 8$ \\
GFRP average & $789 \pm 55$
\end{tabular}

Table 2: Fracture toughness values of T800/913 specimens and E-Glass/913 specimens. 